\title{
The Wide-Field Imaging Interferometry Testbed: Enabling Techniques for High Angular Resolution Astronomy
}

\author{
S. A. Rinehart ${ }^{a}$, T. Armstrong ${ }^{b}$, Bradley J. Frey $^{a}$, J. Jung $^{a}$, J. Kirk $^{c}$, David T. Leisawitz $^{a}$, \\ Douglas B. Leviton ${ }^{a}$, R. Lyon ${ }^{a}$, Stephen Maher ${ }^{d}$, Anthony J. Martino ${ }^{a}$, T. Pauls ${ }^{b}$ \\ ${ }^{a}$ NASA Goddard Space Flight Center \\ ${ }^{b}$ Naval Research Laboratory \\ ${ }^{c}$ Orbital Space Systems \\ ${ }^{d}$ SSAI
}

\begin{abstract}
The Wide-Field Imaging Interferometry Testbed (WIIT) was designed to develop techniques for wide-field of view imaging interferometry, using "double-Fourier" methods. These techniques will be important for a wide range of future spacebased interferometry missions. We have provided simple demonstrations of the methodology already, and continuing development of the testbed will lead to higher data rates, improved data quality, and refined algorithms for image reconstruction. At present, the testbed effort includes five lines of development; automation of the testbed, operation in an improved environment, acquisition of large high-quality datasets, development of image reconstruction algorithms, and analytical modeling of the testbed. We discuss the progress made towards the first four of these goals; the analytical modeling is discussed in a separate paper within this conference.
\end{abstract}

Keywords: Interferometry, Michelson Interferometer, Wide-Field Imaging, Synthesis Imaging, Testbed

\section{INTRODUCTION}

High angular resolution measurements will be critical for the successful exploration of a wide array of NASA's scientific goals, ranging from understanding the origins of stars and planets to the evolution of galaxies. For many applications, a single-aperture telescope sufficient to satisfy the scientific angular resolution requirements is impractical; interferometry provides the only practical alternative. Continued development of ground-based systems and laboratory testbeds provides evidence of the potential power of interferometric techniques.

\subsection{Scientific Motivation}

The Wide-Field Imaging Interfometric Testbed (WIIT) was originally designed to demonstrate a key technology for future far-infrared interferometers ${ }^{1}$. Observations at long wavelengths (i.e. the far-infrared and submillimeter) are critical for a variety of astrophysical investigations. Many objects, such as debris disks, emit most of their light in the FIR. Others, such as star formation regions, are enshrouded by dust that extincts short-wavelength emission. Further, this wavelength regime includes many important diagnostic spectral lines and features. Observations at these long wavelengths with IRAS, ISO, and Spitzer have provided a wealth of new data; however, the utility of these observations has been limited by their lack of angular resolution.

Interferometry provides a powerful tool for obtaining high angular resolution at these long wavelengths. High angular resolution at long wavelengths is critical for observing dust structures within circumstellar disks (e.g. debris disks), for eliminating source confusion in deep-sky images (e.g. the Hubble Deep Field), and for learning how molecular material is distributed around nearby stars. These are compelling scientific issues that can only be addressed by high angular resolution at long wavelengths.

Further author information: (Send correspondence to S.A.R.)

S.A.R.: E-mail: Stephen.A.Rinehart@nasa.gov, Telephone: 1301286 4591, Address: NASA - Goddard Space Flight Center, Mail Code 665, Greenbelt, MD 20771. 


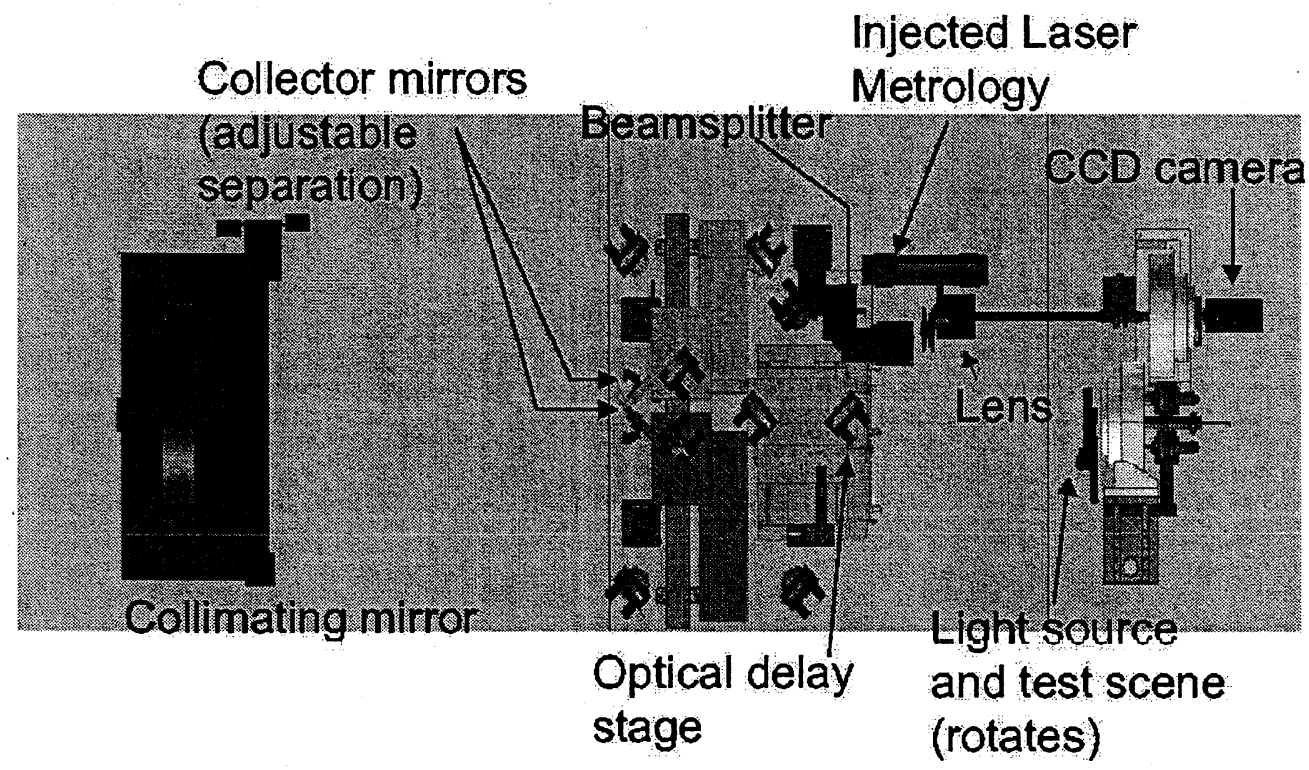

Figure 1. A top view of WIIT generated from a 3-D solid model. In addition to the labelled components, $u-v$ plane coverage is provided by two linear stages upon which the collector mirrors are mounted and by two rotation stages upon which the source scene and camera are mounted.

The original mission concept for which WIIT was developed was the Submillimeter Probe of the Evolution of Cosmic Structure (SPECS) ${ }^{2}$, a 1-km baseline FIR interferometer. SPECS was studied as a NASA Vision Mission. Another concept, studied as a candidate NASA Origins Probe, is the Space Infrared Interferometric Telescope (SPIRIT) ${ }^{3}$. An overview of the SPIRIT design requirements can be found in another paper within this conference ${ }^{4}$; other papers within this conference discuss individual subsystems of SPIRIT, including the optical ${ }^{5}$, mechanical ${ }^{6}$, and thermal design ${ }^{7}$, as well as the detector requirements and options ${ }^{8}$.

\subsection{A Brief History of WIIT}

In the early formulation of FIR interferometric missions, the recognition was made of ability of a space-based interferometer to obtain spectral and spatial information simultaneously. This possibility has been described previously ${ }^{9}$; in this "double-Fourier" mode of operation, the instrument provides a spectrum for every spatial resolution element. A difficulty with this technique, however, is the limited field-of-view of a traditional Michelson interferometer. A standard Michelson interferometer uses a single detector element, with a field-of-view determined by the diffraction limit of the individual collector mirrors $\left(\sim \lambda / D_{t e l}\right)$. In theory, however, one can replace the single detector element with a detector array, allowing one to obtain both spectral and spatial information over a wide field-of-view. WIIT was designed to provide proof-ofconcept of this theory of wide-field imaging interferometry. WIIT also provides a valuable opportunity to study effects of systematics on data quality, and provides real-world data that can be used to test and refine the algorithms and techniques needed for wide-field, double-Fourier interferometry.

Previous papers have provided detail on the development and capabilities of WIIT ${ }^{10,11,12}$. In this paper, we discuss recent developments and the plan for continued research with WIIT. Excluded from this discussion is the WIIT analytical optical system model, which is discussed in another paper at this conference ${ }^{13}$.

\subsection{The Testbed}

WIIT is a 1:150 scale model of the space far-IR interferometer SPIRIT ${ }^{3,14}$. It was built to operate at visible rather than FIR wavelengths for practical reasons: (a) the optical apertures and the delay line are scaled down from those intended for a FIR instrument in proportion to wavelength; (b) a CCD detector with sensitivity limited by photon noise was commercially 


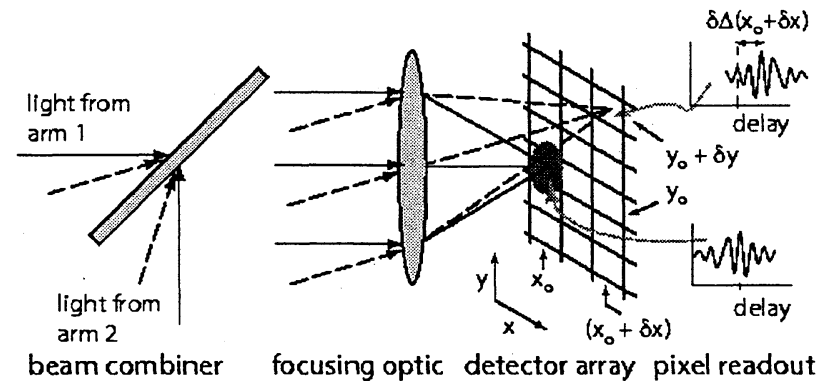

Figure 2. Interference finges from field angles oustide the primary beam (the solid ellipse) can be recorded simultaneously in the separate pixels of the detector array. If light from a source located on the optical axis of the interferometer (solid lines) is focused onto pixel $\left(x_{\circ}, y_{\circ}\right)$, then light from an off-axis source (dashed lines) might reach pixel $\left(x_{\circ}+\delta x, y_{\circ}+\delta y\right)$ after traversing opposite arms of the interferometer. The white light fringe packet in the interferogram from the latter pixel is displaced relative to the interferogram from the on-axis source. The field-of-view is dictated by the number of pixels in the array.

available and inexpensive; (c) other components, such as translation and rotation stages, were available off-the-shelf and inexpensive; and (d) a standard commercial optical metrology system was affordable and accurate to a small fraction of a visible wavelength, an essential requirement for interferometry.

The layout of the testbed is shown in Figure 1; a complete description of the operation of WIIT has been published elsewhere ${ }^{11}$, but we provide a brief overview here. Light from the test scene, located at the focus of the collimating mirror, is projected into the interferometer. The two collector mirrors feed the two arms of the interferometer. One of these arms consists solely of fixed flat mirrors (the "fixed" arm), while the other includes a pair of mirrors mounted on the delay line stage (the "delay" arm). This provides an optical path difference between the two arms of the interferometer. The beams from the two arms are recombined within the beamsplitter, and the output from one of the beamsplitter's output ports is focused onto a CCD camera. The source stage and the camera can be rotated, providing access to all possible baseline orientations, and the collector mirrors can be moved along a straight rail in order to vary baseline length.

The injected metrology system provides differential path length measurements for the two arms of the interferometer. This system provides knowledge of the optical path difference between the two arms of the interferometer to better than $10 \mathrm{~nm}$ for every individual camera exposure. In principle, this system can achieve a measurement accuracy of 1.24 $\mathrm{nm}$; within our development laboratory, we are limited by effects of air turbulence within the room. To understand the impact of the environment on our data, and to monitor how the environment changes over time, WIIT uses several different housekeeping systems. These include an array of temperature sensors located on and around the optical table, optical power monitors, and optical encoders which provide absolute position measurement of the different rotation and translation stages.

The novel aspect of WIIT is the use of a detector array (a CCD camera, in this case) instead of the single-pixel detector used in a traditional Michelson interferometer. As shown schematically in Figure 2, each pixel on the detector records light arriving from a different part of the sky (test scene). As the delay line is scanned, each pixel records an interferogram unique to the field-of-view of the pixel. By using observations at a wide range of $u$-v points, reconstructed images can be produced for each pixel; these images can then be mosaiced together, producing a wide field-of-view image with the full interferometric angular resolution. By providing a long delay line scan, we are further able to obtain spectral information for the observed sources; to obtain a resolution $R$ requires an optical path delay scan range of $\delta=R \lambda$.

The one complication of this scheme is that the interferometric fringes have different relative phases for the different detector pixels, due to the geometric optical delay inherent for sources off the axis of the interferometer. In order, therefore, to obtain data on the full fringe packet for each point within the full field-of-view, we require a optical delay stroke larger than the geometric optical delay. However, such long optical delays are already required in order to achieve moderate spectral resolution. As a concrete illustration, let us consider a hypothetical observations with a spectral resolution of $R=1000$, a baseline length of $b=36 \mathrm{~m}$, a wavelength of $100 \mu \mathrm{m}$, and a field-of-view of $\theta=1 \mathrm{arcmin}$. In order to obtain the desired spectral resolution, an optical scan distance of $R \lambda=100 \mathrm{~mm}$ is required. To obtain the wide field-of-view, 
we require an optical scan length of $b \sin \theta=10.4 \mathrm{~mm}$. In this regime, scanning of the optical delay line simultaneously fulfills the needs for both spectroscopy and the wide field of view.

\section{RECENT PROGRESS}

\subsection{Automation}

One of the major milestones for WIIT is the complete automation of the system. Full automation is a complicated problem, involving a number of challenging aspects. As a few examples, the testbed needs to: continuously monitor the positions of all the translation and rotation stages; autonomously align itself between different u-v points; and capture all of the collected data, including housekeeping information, Originally, this level of automation was considered important in order to allow operation within carefully controlled environments. This requirement has been accentuated with the current move into the Advanced Interferometry and Metrology (AIM) Laboratory (discussed below). The AIM Laboratory is a clean facility, with very tight environmental controls; the greatest risk to cleanliness of the facility is the presence of people within the laboratory. Likewise, people are the single-largest contributor to environmental disturbances. In order to take full advantage of this new facility, we must minimize the amount of user activity required within the laboratory. In addition, the time required to complete a single set of observations makes automation a necessity. A Nyquist-sampled set of $u-v$ data for a single target can require over 48 hours of continuous observations; dedicated manning of the testbed for such long periods is impractical. By automating the system, the operator will be able to program a complete set of testbed observations for a given source. Once under way, the operator can leave, returning only when the observation is complete - the automated systems allows nearly-continuous data acquisition of data.

Significant progress has already been made on automation of the testbed. To simplify discussion, the automated system can be thought of separate hardware and software components, even though these elements are intertwined. Recently, we have added the two final hardware components needed for full automation of the testbed: two shutters and a tip/tilt mirror. These components are designed to allow autonomous alignment of the system. Due to imperfections in the baseline mirror guide rail, as well as due to time-varying environmental conditions, the relative alignment of the two arms can be lost over time. We can, however, self-align by making use of these components while observing a point source with the testbed. By alternately closing the two shutters, we are able to precisely measure the positions of the source (from the light passing through each of the two arms of the interferometer). The relative offset of the two arms of the interferometer can then be precisely determined, and the tip/tilt mirror mount can be adjusted to bring them back into alignment. This procedure can be repeated in order to verify the alignment. We have not yet measured the accuracy of the alignment possible with this system, but believe that it should be as good or better than achievable manually, and that it will be highly repeatable.

The shutters also provide the ability to easily obtain calibration data without requiring the operator to enter the clean room. At the simplest level, by closing both of the shutters, we ensure that no light passes through the two arms of the interferometer. This allows us to measure the stray light on the detector array. We can also use the shutters to measure the illumination profile on the synthetic aperture. By closing off one shutter, we measure the relative flux entering the other arm of the interferometer. By repeating this measurement at multiple baselines, for both arms of the interferometer, we can accurately determine the illumination profile. We have carried out such calibration measurements with WIIT previously, but by making use of the automated components we will be able to incorporate calibration directly into the data acquisition scheme. This will provide an opportunity for monitoring the testbed behavior as part of standard observational routine, and for assessing the quality of incoming data.

In addition, we are developing new control software for WIIT. The Advanced Architectures and Automations Branch (Code 588) at NASA Goddard has developed the Instrument Remote Control (IRC) environment. The IRC is a robust tool which allows for distributed control and monitoring of remote instruments ${ }^{16}$. The software is configurable, so that it is easily modified and extended for use by a variety of research programs. Built into the IRC are the tools needed to enable the complex array of communications between different hardware elements of the testbed, as well as the software tools for quickly and clearly displaying data from the testbed in a variety of different forms. By working with the IRC, we take advantage of both existing tools at GSFC and existing expertise with their use and development.

\subsection{Improved Environment}

At present, we are moving WIIT into the Advanced Interferometry and Metrology (AIM) Laboratory at Goddard, a new facility completed in January 2007. The AIM Laboratory was developed specifically to enable interferometric testbed 


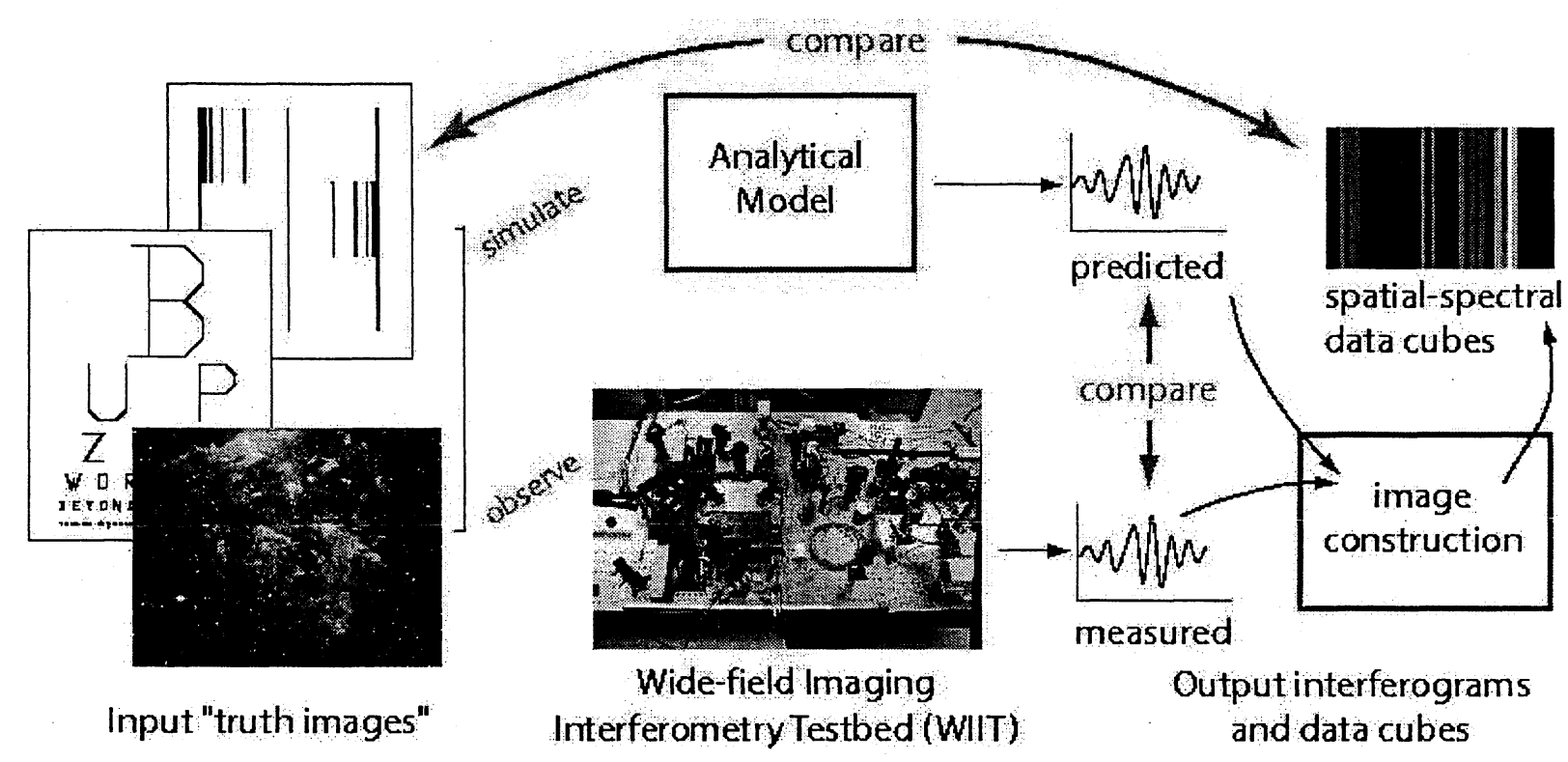

Figure 3. In future experiments, we will combine data from the testbed with results of analytical modeling to learn how best to synthesize spatial-spectral datacubes from wide field double Fourier data.

research, by providing a clean facility with excellent environmental control. The Laboratory is a clean facility, controlled to class 10000; air handling is a low velocity gravity airflow system, limiting air turbulence within the room. The first characterization test of this facility demonstrated $1 \mathrm{~nm}$ rms wavefront stability over an unbaffled 10 meter path length. The facility has thermal control designed to provide temperature stability to better than $\pm 0.5^{\circ} \mathrm{C}$, and humidity to $\pm 5 \%$; tests in the laboratory have shown that it exceeds these requirements. Acoustic isolation of the lab is provided by sandfilled masonry walls with acoustically absorbant panelling. The Lab is decoupled from the existing building's structure frame, roof, and external sheathing, providing a high degree of vibration isolation. A second stage of isolation is provided by active control isolation systems on the individual optical tables. Preliminary tests have shown vibration stability with $20-40 \mathrm{~dB}$ of damping from the quiet floor to the actively controlled tables over the range 1-1000 Hz.

The AIM Laboratory offers a vastly superior environment than the WIIT development laboratory. While the optical path length of the two arms of WIIT are only $\sim 2$ meters, the measured path length fluctuations in the environment of our development laboratory have been measured at $\sim 30 \mathrm{~nm}$ rms. By moving WIIT into the AIM lab, and taking advantage of the superior environment, the uncertainty in fringe visibility will be reduced from about $11 \%$ to $1-2 \%$ (depending upon the source flux in the camera) ${ }^{15}$. The reduced phase noise will enable us to construct much higher quality images, and in this improved environment the data quality will be limited by the interferometer rather than the environment. This is a qualitatively different operational regime which has not been explored previously, and which must be explored in order to address real issues confronting future spaceborne interferometers.

\subsection{Data Acquisition}

As we have continued acquiring data with WIIT, our methodology has been refined in order to obtain maximum value from the data. Our approach is shown schematically in Figure 3. For a given test scene (one of the images on our microlithographed source plate), we both observe the source directly with the testbed and produce simulated interferometric data sets. As a first verification of data quality, we compare the interferograms obtained with the testbed to the simulated data. This comparison allows us to understand the sources of uncertainty and error within the testbed data, and provides a check on overall data quality. Following this comparision, we reconstruct the source images from the interferometric data from both WIIT and our simulations. The reconstructed images are then compared directly to the source images. This comparison, including the examination of the reconstructed spectrum against the input spectrum, provides an assess- 

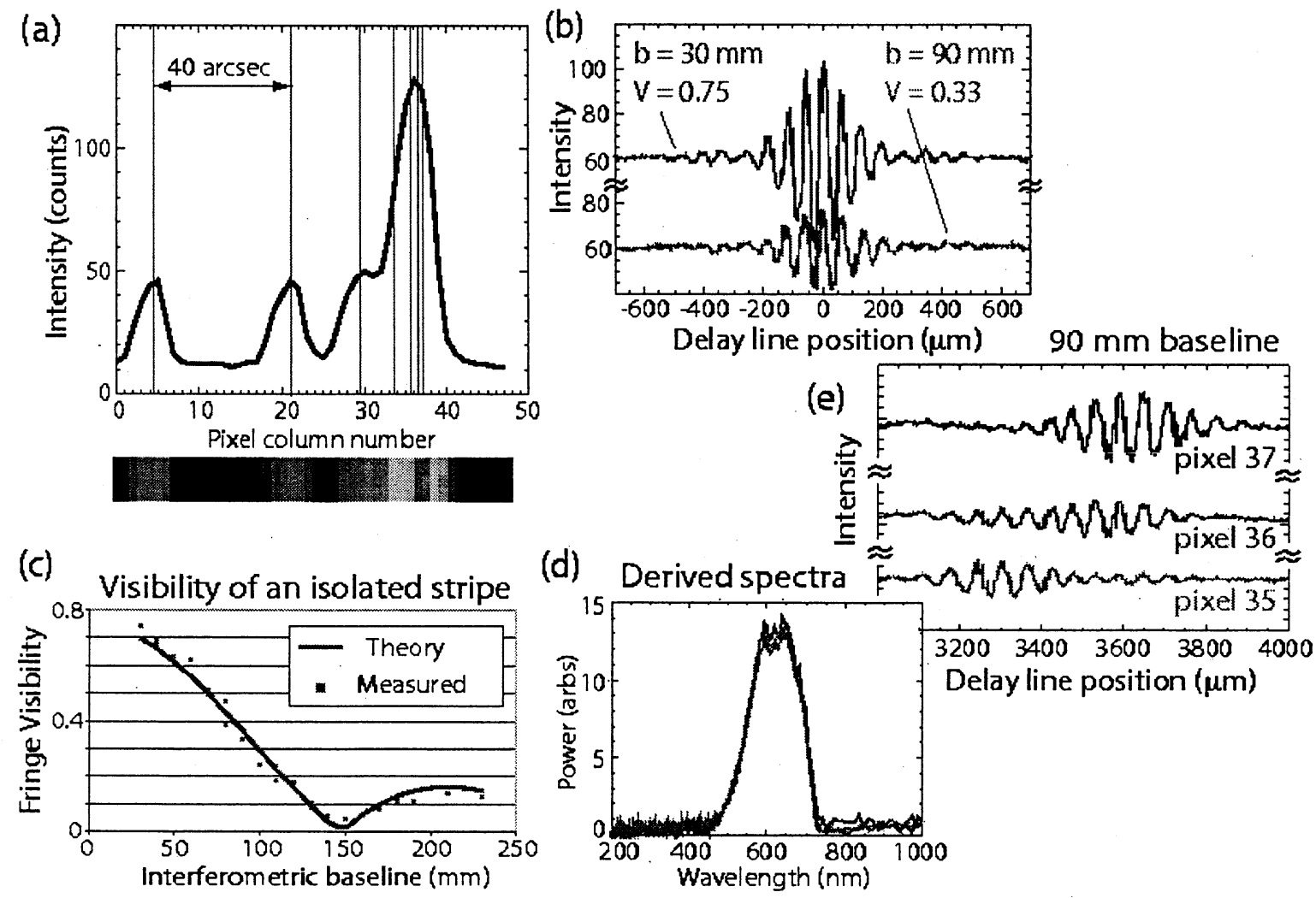

Figure 4. We have acquired a large amount of data with WIIT within our development laboratory. In panel (a), we plot a horizontal cut of the test scene illumination across the detector array (a subarray of the actual image is shown bewlow the plot). In (b), we show the interferograms obtained for an isolated source in the test scene at two different baselines. By measuring these interferograms at multiple baselines, we obtain the visibility function (c) which provides the information for spatial image reconstruction. By Fourier transforming the fringe patterns, we obtain the spectrum of the source (d). Finally, in panel (e), we show how one resolves multiple sources with small seperation.

ment of how data quality affects final data products. Further, these comparisions are critical for testing and evaluating algorithms.

In preparation for moving into the AIM Laboratory, we have temporarily ceased acquisition of new data. Instead, we have been carefully planning upcoming observations to take maximum advantage of our time within the improved environment. We anticipate operating WIIT within the AIM Lab for a period of approximately two years, during which time we will acquire a vast amount of data for study*. In Section 3 we discuss some of the planned observations in more detail.

\subsection{Algorithms}

The data acquired with WIIT to date have been very valuable, and have led to the first demonstration of wide-field imaging interferometry. Figure 4 shows some representative data from the testbed. The test scene consists of a series of seven stripes, located at the positions of the vertical lines in Figure 4a. These stripes are oriented perpendicular to the baseline. The leftmost pair of stripes are separated by $40 \mathrm{arcsec}$; each subsequent pair of stripes has a separation reduced by a factor of two, down to the 1.25 arcsec separation of the rightmost pair. The solid black line in Figure 4a shows the overall

\footnotetext{
${ }^{*}$ We plan on making all data acquired with WIIT available to the scientific community. Please contact the authors of this paper if you are interested in working with the data.
} 


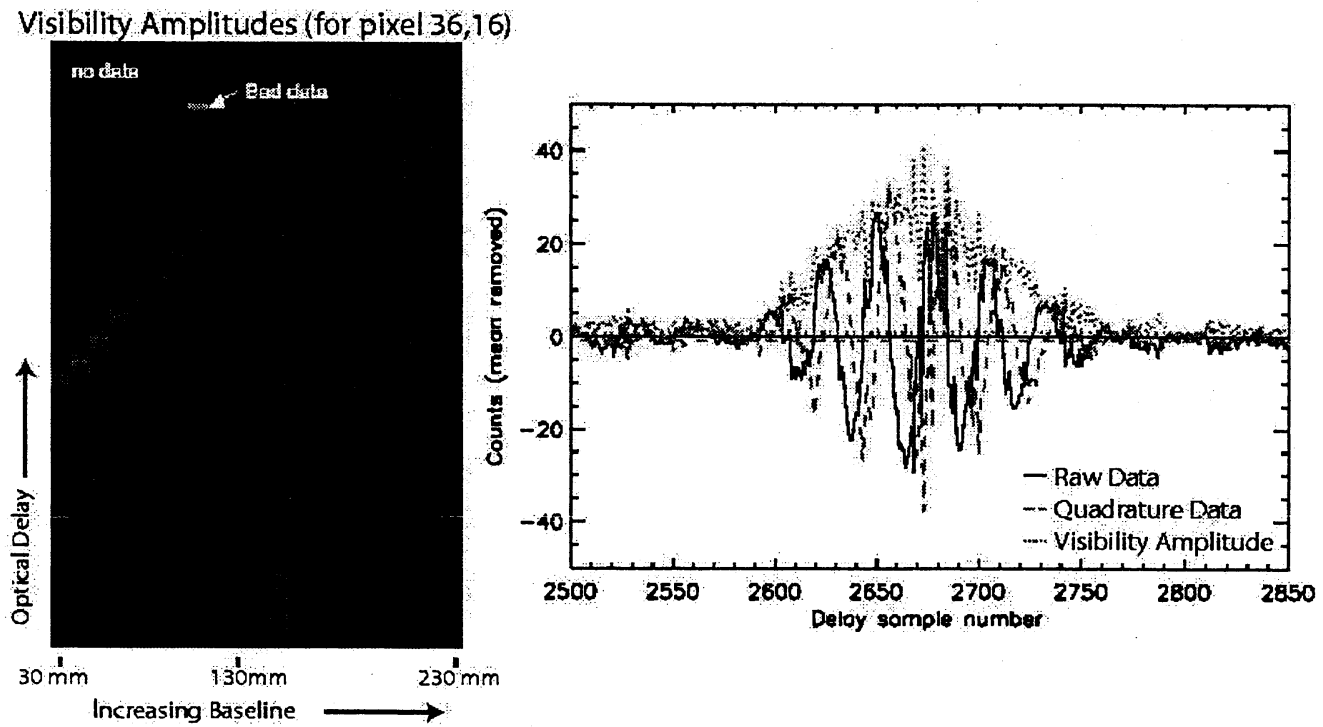

Figure 5. The image at left displays the change in the relative phase of the interferogram as a function of baseline. The columns in the image correspond to increasing baseline distance, while the vertical axis represents the position in optical delay space. From this data, the quadrature signal can be measured from the data using a Hilbert transform. The visibility amplitude can then be calculated. The Hilbert transform simultaneously provides visibility phase information, which is needed for accurate image reconstruction.

illumination pattern on the detector array, and we see clearly the blending of the unresolved sources on the right hand side. Figure $4 \mathrm{~b}$ shows the interferograms obtained for two different baselines for the leftmost (isolated) line; we see that because the individual line is resolved by the interferometer, the visibility is decreasing. Such visibility measurements at many baselines allow us to determine the spatial structure of the source (Figure 4c). The observed visibility function corresponds to an 0.85 arcsec stripe; this is the known size of the source. Similarly, if we Fourier transform the fringe packets as shown in Figure 4b, we obtain the spectrum of the source (Figure 4d). The observed spectrum is consistent with expectations, based upon measurements of the properties of the components of the testbed.

The advantage of interferometry is the ability to resolve closely-spaced sources. There are three seperate stripes contained in pixels 35-37. Figure 4e demonstrates clearly how these sources are resolved. As we observe fringes at longer baselines, the relative optical delay between the fringe packets of different sources increases. As we see in Figure 4e, the two fringe packets separated by $\sim 280 \mathrm{~nm}$ of delay are distinguishable in pixels 35 and 36 , the expected signature of two stripes separated by 1.25 arcsec and observed with a baseline of $90 \mathrm{~mm}$. The fringe packets in this plot appear somewhat distorted; while the spectra of the two stripes are identical, the small separation between their fringe packets leads to blending of the interference fringes. By observing the separation between fringe packets from different sources within a scene as a function of baseline, we derive the angular separation between the individual sources. In fact, it is possible in principle to use the entire image to provide the phase information needed for image reconstruction.

The above description provides a relatively succinct explanation of how one derives both spatial and spectral information from the interferometric data obtained with WIIT; it is also an accurate depiction of the simplest possible algorithmic approach to image reconstruction. With a complete set of data, a "brute force" approach making use of large numbers of Fourier transforms can lead to a high fidelity image reconstruction. However, this brute force approach is very computationally intensive, and it not necessarily robust when confronted with noise and uncertainty in the data. As part of our research effort, we have explored other image reconstruction algorithms, searching for computationally efficient techniques which lead to very high fidelity image reconstruction. One of the most promising of these makes use of the Hilbert transform.

In Figure 5, we show some recent results using our Hilbert transform method. The image is a representation of the 
interferograms as a function of baseline. As the baseline length increases (from left to right in the image), we see the fringe packet moving up in the image (increasing in optical delay space). This shows clearly the presence of an off-axis source. The peaks at longer baselines are difficult to see here, as the individual sources are resolved by the interferometer and their visibility drops dramatically. By applying the Hilbert transform to this data, we are able to derive the visibility amplitudes and visibility phases. The inverse Fourier transform of the visibility amplitude gives the spectrum of the spatial mode of the source specific to the particular $u-v$ point (baseline). The visibility phase gives the phase shift in that Fourier component of the image. This, in combination with the visibility amplitude, provides the complex visibility function, which can be transformed into the image and its spectrum for each spatial resolution element.

\section{FUTURE DIRECTIONS}

Here we detail some of the future directions for the WIIT testbed. In addition to the work discussed below, the next few years will witness significant development of our analytic models of the testbed. These efforts are discussed in a companion paper within these proceedings ${ }^{13}$.

\subsection{Near-term objectives}

At present, there are several active areas of development around WIIT. The largest effort at present is focused on moving the testbed into the recently-completed AIM Laboratory. As discussed previously, by moving into this state-of-the-art facility, we will be able to acquire data representative of those obtainable with an interferometer in space, in which the instrument, rather than the environment, limits data quality. Preparations for this move have been nearly completed, and we anticipate having WIIT operational within the AIM Laboratory by November 2007.

By the end of this year, we will have also completed the automation of the testbed. As described previously, we have made significant progress towards this milestone; over the next few months we will combine the different aspects of testbed automation. This will make the testbed fully functional via remote control. This capability will allow us to take data at a very high cadence, without requiring the presence of or intervention by operators, leading to the production of large amounts of high quality data. Further, by obviating the need for human intervention with the testbed, we will be able to leave the testbed undisturbed within the AIM Laboratory. This will help ensure the highest level of environmental stability, and therefore the highest quality of data possible.

With WIIT fully operational within the AIM Laboratory, we will recharacterize the testbed. Within the development laboratory, we have explored a large number of factors which can lead to degradation of data from WIIT. These factors can arise from external sources (such as air turbulence) or from the testbed itself (delay line jitter). With the help of the WIIT model, we will isolate the individual sources of noise, and determine their individual impacts on the data. Many of the fringe-reducing effects (optical surface imperfections) can be compensated for via calibration of the final data; the majority of factors which cannot be mitigated via calibration are environemental (air turbulence), and will be greatly reduced by operation in the AIM Laboratory. We will produce an accurate error budget for the testbed and use it to calibrate the testbed. Using a few simple calibration measurements within our automated operation (stray light observations, focus verification, measurement of the illumination profile, etc.), we will monitor data quality from WIIT, as well as the overall health of the testbed.

\subsection{Mid-term objectives}

As part of the recharacterization effort with WIIT, we will acquire data for a few simple sources. In addition to being valuable for characterization and calibration, this will allow us to test our overall methodology. This will signal the start of our high data cadence program. The first stage of our observing program will cover about 20 wide-field scenes, ranging dramatically in both spatial and spectral complexity. For each of these sources, we will densely oversample both the $u-v$ plane and the fringe packet (over a range including at least a few hundred fringes). Full sets of data for simple sources will provide a clear demonstration of the capabilities of WIIT, while verifying the performance of our spectral-spatial image construction techniques. We will progressively advance to scenes of increasing complexity, refining our algorithmic approach. This will demonstrate and assess the techniques needed for wide-field imaging interferometry. Our data sets will oversampe all three dimensions of the data (the $u \cdot v$ plane and optical delay). By choosing particular subsets of the data, we will be able to explore how different sampling methods impact data quality. 
Another major component of the WIIT program is the continuing development of algorithms for image reconstruction. As discussed previously, we have made significant progress using a Hilbert transform method. As we move forward, we will continue working with this technique, further refining the algorithm. Further, we plan to develop an aggregate method for using the Hilbert transform method with the large sets of data that WIIT will produce. Concurrent with this development, we will explore image reconstruction using direct transformation methods. Finally, as part of the algorithm development progam, we will use Maximum Entropy Method algorithms for use with optical/infrared interferometry. Such algorithms are already rather well-developed, but we will be exploring their optimization for use with our Hilbert transform tools. On longer timescales, we will build upon our experience with the Hilbert transform to study the applicability of Hilbert-Huang methods. We will also explore image reconstruction using model parameter optimization techniques.

\subsection{Long-term objectives}

The first period of data acquisition, discussed above, will make use of the "step-and-stare" mode. In this mode, the baseline mirrors are moved to a fixed position and locked in place; while they are locked, the delay line is stroked to provide fringes on the detector. This observation mode is the simplest possible; however, it is not analagous to the operational modes for future space-based interferometers. Such interferometers could be rotating continuously during operation. We will therefore study an "on-the-fly" observing mode, a mode analagous to envisaged spacecraft operations, for the second phase of data acquisition. In this mode, data (fringes) are acquired while the baseline mirrors are in motion. This leads to some smearing of the fringes as the baseline positions change during a single delay line scan. Implementation of this mode will be rather straightforward, as it is based upon the testbed automation effort that is nearly complete. We plan on using the on-the-fly observing mode for the same set of 20 sources observed during the first data acquisition period. This plan may evolve as we work with the testbed further.

As mentioned previously, all of our data acquisition will have dense sampling in all three dimensions of the interferometric datacube. We will use this sampling to study potential optimization techniques for $u-v$ coverage. It is often assumed that the best way to ensure a high-quality reconstructed image is to Nyquist sample the $u-v$ plane. Such sampling yields a synthetic point spread function (PSF) that is most similar to the PSF of a single aperture telescope. However, such coverage requires a large number of individual observations, and it may be possible to optimize the coverage to reduce the number of observations while still maintaining good image fidelity. Further, for some sources, it may be advantageous to tailor the $u-v$ coverage to the object of interest. We will explore these issues both analytically and by using WIIT data.

\section{CONCLUSIONS}

We have made significant progress with WIIT in the past year, and are on the cusp of producing a wealth of high quality interferometric data. These data will be representative of those obtainable with a doulbe Fourier interferometer in space. The new data and the WIIT analytical model will allow us to explore practical aspects of space interferometry. Ultimately, we will apply these lessons directly to future spaceborne interferometers such as SPIRIT, SPECS, and TPF-I, as well as to potential spaceborne interferometers for earth-observation (EASI) or observations of Mars (L2-MAP).

\section{ACKNOWLEDGMENTS}

Funding for WIIT is provided by NASA Headquarters through the ROSS/APRA Program and by the Goddard Space Flight Center through its IR\&D program.

\section{REFERENCES}

1. Feinberg, L. Leisawitz, D., Leviton, D.B., Zhang, X., \& Lyon, R. 2000. In Imaging Spectrometry VI, Proc. SPIE 4132, 365 .

2. Rinehart, S.A., 2006. In Space Telescopes and Instrumentation I, Proc. SPIE 6265, 60.

3. Leisawitz, D., Baker, C., Barger, A., Benford, D., Blain, A., Boyle, R., Broderick, R., Budinoff, J., Carpenter, J., Caverly, R., Chen, P., Cooley, S., Cottingham, C., Crooke, J., DiPietro, D., DiPirro, M., Femiano, M., Ferrer, A., Fischer, J., Gardner, J.P., Hallock, L., Harris, K., Hartman, K., Harwit, M., Hillenbrand, L., Hyde, T., Jones, A., Kellogg, J., 
Kogut, A., Kuchner, M., Lawson, W., Lecha, J., Lecha, M., Mainzer, A., Mannion, J., Martino, A., Mason, P., Mather, J.C., McDonald, G., Mills, R., Mundy, L., Ollendorf, S., Pellicciotti, J., Quinn, D., Rhee, K., Rinehart, S.A., Sauerwine, T., Silverberg, R.F., Smith, T., Stacey, G., Stahl, H.P., Staguhn, J., Tompkins, S., Tveekrem, J., Wall, S., and Wilson, M., 2007. "The Space Infrared Interferometric Telescope (SPIRIT): High-resolution imaging and spectroscopy in the far-infrared", J. Adv. Sp. Res., in press, doi:10.1016/j.asr.2007.05.081.

4. Hyde, T.T., Leisawitz, D.T., and Rinehart, S.A., 2007. "System engineering the Space Infrared Interferometric Telescope (SPIRIT)", Proc. SPIE 6687, this volume.

5. Wilson, M.E., Leisawitz, D.T., Martino, A.J., Rinehart, S.A., Crooke, J.A., Tveekrem, J.L., Budinoff, J.G., Quijada, M.A., and Hyde, T.T., 2007. "The Space Infrared Interferometric Telescope (SPIRIT): Optical system design considerations", Proc. SPIE 6687, this volume.

6. Budinoff, J.G., Leisawitz, D.T., Rinehart, S.A., DiPirro, M.J., Jones, D.L., Hyde, T.T., and Taylor, B., 2007. "Mechanical design of the Space Infrared Interferometric Telescope (SPIRIT)", Proc. SPIE 6687, this volume.

7. DiPirro, M.J., Cottingham, C., Boyle, R., Ollendorf, S., and Leisawitz, D.T., 2007. “The SPIRIT thermal system”, Proc. SPIE 6687, this volume.

8. Benford, D.J., Rinehart, S.A., Hyde, T.T., and Leisawitz, D.T., 2007. "Cryogenic far-infrared detectors for the Space Infrared Interferometric Telescope (SPIRIT)", Proc. SPIE 6687, this volume.

9. Mariotti, J.-M. \& Ridgeway, S.T. 1988, A\&A 195, 350.

10. Rinehart, S.A., Armstrong, T., Frey, B., Jung, J, Kirk, J., Leisawitz, D.T., Leviton, D., Lyon, R., Martino, A., Mundy, L.G., Pauls, T., \& Thompson, A. 2006. In Advances in Stellar Interferometry, Proc SPIE 6268, 100.

11. Leisawitz, D.T., Frey, B.J., Leviton, D.B., Martino, A.J, Maynard, W.L, Mundy, L.G, Rinehart, S.A., Teng, S.H., \& Zhang, X. 2002. In Interferometry in Space, Proc. SPIE 4852, 255.

12. Rinehart, S.A., Frey, B.J., Leisawitz, D.T, Leviton, D.B., Martino, A.J, Maynard, W.L, Mundy, L.G, Teng, S.H., \& Zhang, X. 2002. In Interferometry in Space, Proc. SPIE 4852, 674.

13. Martino, A.J., Leisawitz, D.T., Thompson, A.K., Rinehart, S.A., and Frey, B.J., 2007. An optical model of the Wide-field Imaging Interferometry Testbed, Proc. SPIE 6687, this volume.

14. Leisawitz, et al. 2004. In Space Telescopes and Instrumentation I, Proc. SPIE 6265, 120.

15. Rinehart, S.A., Armstrong, T., Frey, B., Kirk, J., Leisawitz, D.T., Leviton, D., Lobsinger, L., Lyon, R., Martino, A., Mundy, L.G., Pauls, T., \& Sears, E. 2004. In New Frontiers in Stellar Interferometry, Proc SPIE 5491, 1790.

16. Ames, T., Koons, L,. Sall, K., \& Warsaw, C. 2000. In Advanced Telescope and Instrumentation Control Software, Proc. SPIE 4009, 2. 